Revue internationale P.M.E.

Économie et gestion de la petite et moyenne entreprise

\title{
Gouvernement territorial et district industriel : l'exemple de Montpellier
}

\section{Colette Fourcade}

Volume 6, numéro 1, 1993

URI : https://id.erudit.org/iderudit/1008166ar

DOI : https://doi.org/10.7202/1008166ar

Aller au sommaire du numéro

Éditeur(s)

Presses de l’Université du Québec

ISSN

0776-5436 (imprimé)

1918-9699 (numérique)

Découvrir la revue

Citer cette note

Fourcade, C. (1993). Gouvernement territorial et district industriel : l'exemple de Montpellier. Revue internationale P.M.E., 6(1), 101-121.

https://doi.org/10.7202/1008166ar
Résumé de l'article

La politique interventionniste des gouvernements territoriaux peut-elle contribuer à la création d'un district industriel ? Le district Industriel peut en effet apparaître comme un mode d'organisation efficient d'un système productif local ; aux yeux des responsables des politiques de développement local, la construction d'une telle organisation constituerait l'objectif visé. Il convient toutefois de ne pas considérer le district industriel comme un modèle de développement local, mais bien comme un mode particulier d'organisation de systèmes localisés de petites entreprises. En revanche, traité en tant que concept de référence, il apparaît alors comme un outil d'analyse pertinent pour la compréhension de la structuration et de l'évolution de tels systèmes. C'est donc comme outil analytique qu'il est utilisé pour estimer l'impact de la politique d’intervention menée par les autorités locales dans l'aire de Montpellier, France. 


\title{
Gouvernement territorial et district industriel: l'exemple de Montpellier
}

Colette FOURCADE*

Université Montpellier I

\begin{abstract}
RÉSUMÉ
La politique interventionniste des gouvernements territoriaux peut-elle contribuer à la création d'un district industriel ? Le district industriel peut en effet apparaître comme un mode d'organisation efficient d'un système productif local; aux yeux des responsables des politiques de développement local, la construction d'une telle organisation constituerait l'objectif visé.

II convient toutefois de ne pas considérer le district industriel comme un modèle de développement local, mais bien comme un mode particulier d'organisation de systèmes localisés de petites entreprises. En revanche, traité en tant que concept de référence, il apparaît alors comme un outil d'analyse pertinent pour la compréhension de la structuration et de l'évolution de tels systèmes. C'est donc comme outil analytique qu'il est utilisé pour estimer l'impact de la politique d'intervention menée par les autorités locales dans l'aire de Montpellier, France.
\end{abstract}

\section{ABSTRACT}

Are interventionnal policies led by the local authorities able to contribute and create an industrial district? The industrial district seems to be an efficient mean in the organization of a local industrial system. For the people who are in charge of the local development policies, the building of such an organization constitutes the target. Actually, the industrial district must not be viewed as an idealistic model of development, but as a particular pattern in the organization of local small business systems. But taken as a referential concept, it seems to be an efficient analytic implement to understand the structuration and the evolution of such systems. It is the reason why it is used here as an analytical implement to estimate the impact of the interventionnal policies led by the local government inside the Montpellier area, France.

* Colette Fourcade est Docteure d'État en sciences économiques, maître de Conférences à l'Université Montpellier I. Membre fondateur de l'Équipe de recherche sur la firme et l'industrie, ses recherches portent sur la petite entreprise en relation avec le développement local. Adresse : E.R.F.I., 39 rue de l'Université, 34060 Montpellier, Cedex, France. 


\section{RESUMEN}

La politica intervencionista de los gobiernos territoriales puede e contribuir a la creación de un distrito industrial ? El distrito industrial puede en efecto aparecer como un modo de organización eficiente de un sistema productivo local ; a los ojos de los responsables de las politicas de desarrollo local, la construcción de tal organización constituiría el objetivo deseado.

Sin embarbo es conveniente de no considerar el distrito industriel como un modelo de desarrollo local, pero si (como un modo particular de organización de sistemas localizados de pequeñas empresas. En revancha, tratado en calidad de concepto de referencia, el aparece en tal caso como un util de análisis pertinente por la compresión de la estructuración y de la evolución de tales sistemas. De esta manera uno puede utilizarlo como un util analítico por estimar el impacto de la política de intervención llevadas a cabo por las autoridades locales dentro del territorio de Montpellier, Francia.

\section{Introduction}

La politique d'intervention menée par les autorités locales peut-elle contribuer à la création d'un district industriel ? Cette question n'est pas sans intérêt au moment où un certain nombre de territoires se trouvent en quelque sorte « contraints » au développement. Cette contrainte ressort de deux origines : la première peut-être analysée comme la conséquence de la stratégie des grandes entreprises notamment en termes d'externalisation de leurs activités : d'où une modification à la fois du rapport des grandes firmes au local et de leur rapport aux petites entreprises. La seconde s'inscrit, tout particulièrement en France, dans le mouvement de décentralisation des décisions en matière de politique de développement du territoire. Les gouvernements territoriaux peuvent-ils dès lors espérer organiser le développement local en visant à la constitution de districts industriels ? Ce sont quelques éléments de réponse que nous tenterons de fournir en nous appuyant sur l'exemple de l'aire de Montpellier, en France. Au préalable, le concept de district industriel devra être précisé, en distinguant notamment les divers types de districts. Mais au-delà de l'aspect descriptif attaché au district industriel, il conviendra de s'interroger sur l'utilisation du concept dans l'approche du développement local. Ceci nous conduira à évaluer les possibilités de voir émerger une telle organisation en tant que résultante de la politique de développement local.

\section{Le district industriel : concept ou modèle ?}

Tout d'abord, on peut définir un district industriel comme un ensemble de petites unités liées par des relations d'échanges productifs, spécialisées dans une activité 
industrielle donnée et localisées sur un territoire délimité. La notion de district industriel implique donc trois éléments :

- un ensemble de firmes, plutôt de petite taille ;

- un ensemble de relations spécifiques, mais de natures diverses, aussi bien économiques que sociales ;

- un ensemble localisé : la référence à une aire limitée apparaît essentielle, la question d'une délimitation économique et/ou géographique restant posée.

Il convient en premier lieu de préciser le concept, soulignant ainsi la diversité des réalités couvertes par la notion de district industriel; nous apprécierons ensuite la pertinence de ce concept.

\subsection{Du concept de district industriel}

Le cadre conceptuel le plus large pour comprendre la notion de district industriel est indubitablement dérivé d'Alfred Marshall (Zeitlin,1990). Celui-ci estime que des économies externes peuvent être obtenues par la concentration d'un grand nombre de petites firmes œuvrant dans un même secteur industriel - y compris des industries qualifiées d' « auxiliaires », comme la production de machines -, dans une aire géographiquement délimitée. Ce concept marshallien a été réutilisé au début des années 70 pour l'interprétation du développement des régions du centre et du nord-est de l'Italie, appuyé sur des ensembles de petites entreprises.

Ainsi, en se plaçant du point de vue de l'évolution historique récente des districts, S. Brusco (1988) propose un classement selon quatre stades évolutifs :

- le modèle de l'artisan traditionnel : années 50 ;

- le modèle du sous-traitant dépendant : fin des années 60 ;

- le modèle de district industriel $1:$ milieu des années 70;

- le modèle de district industriel 2 : années 80.

La lecture du tableau 1 permet d'effectuer un clivage entre d'une part un ensemble inorganisé de petites entreprises et d'autre part, le district industriel, où l'unité d'analyse n'est plus la grande entreprise, ni les interactions grande entreprise - petites entreprises, mais bien un ensemble géographiquement localisé, caractérisé par un grand nombre de firmes de petite, voire de très petite taille, qui sont impliquées aux différents stades de la production d'un produit homogène, et de manières diverses (Pyke et Sengenberger, 1990). Chaque district apparaît spécialisé dans un produit donné, avec des degrés divers de complexité. 
TABLEAU 1

Évolution des districts : perspective historique

\begin{tabular}{|c|c|c|c|c|}
\hline Critères & $\begin{array}{l}\text { Artisan } \\
\text { traditionnel }\end{array}$ & $\begin{array}{l}\text { Sous-traitant } \\
\text { dépendant }\end{array}$ & $\begin{array}{l}\text { District } \\
\text { Industriel I }\end{array}$ & $\begin{array}{l}\text { District } \\
\text { Industriel II }\end{array}$ \\
\hline $\begin{array}{l}\text { Unité } \\
\text { d'analyse }\end{array}$ & $\begin{array}{l}\text { Petites } \\
\text { entreprises }\end{array}$ & $\begin{array}{l}\text { Petites } \\
\text { entreprises }\end{array}$ & $\begin{array}{l}\text { Ensemble } \\
\text { de firmes reliées } \\
\text { dans une aire limitée }\end{array}$ & \multirow[t]{2}{*}{$\begin{array}{l}\text { Mêmes caractéristiques } \\
\text { que District Industriel I }\end{array}$} \\
\hline Produit & $\begin{array}{l}\text { Produit identique } \\
\text { pour petite et } \\
\text { grande entreprise }\end{array}$ & $\begin{array}{l}\text { Produit différent } \\
\text { entre petite et } \\
\text { grande entreprises }\end{array}$ & $\begin{array}{l}\text { Final } \\
\text { et intermédiaire }\end{array}$ & \\
\hline $\begin{array}{l}\text { Aire } \\
\text { de marche }\end{array}$ & Local & National & $\begin{array}{l}\text { National/ } \\
\text { international }\end{array}$ & \multirow{3}{*}{$\begin{array}{l}\text { Nécessité } \\
\text { de l'interventionnisme } \\
\text { pour accélérer l'introduction } \\
\text { et la diffusion } \\
\text { des nouvelles technologies }\end{array}$} \\
\hline Aptitudes & $\begin{array}{l}\text { Faible productivité } \\
\text { Techniques } \\
\text { de travail } \\
\text { intensif }\end{array}$ & $\begin{array}{l}\text { Inefficacité } \\
\text { par rapport } \\
\text { aux grandes firmes }\end{array}$ & $\begin{array}{l}\text { Innovation } \\
\text { et savoir-faire } \\
\text { par les relations } \\
\text { donneurs d'ordres/ } \\
\text { sous-traitants }\end{array}$ & \\
\hline Technologie & $\begin{array}{l}\text { Faible } \\
\text { Aptitudes acquises } \\
\text { par apprentissage }\end{array}$ & $\begin{array}{l}\text { Faible } \\
\text { Mais certaines } \\
\text { petites entreprises } \\
\text { efficaces }\end{array}$ & & \\
\hline $\begin{array}{l}\text { Échanges inter- } \\
\text { Industriels }\end{array}$ & $\begin{array}{l}\text { Concurrence } \\
\text { imparfaite }\end{array}$ & $\begin{array}{l}\text { Concurrence } \\
\text { oligopsonique }\end{array}$ & $\begin{array}{l}\text { Comportement } \\
\text { de filière }\end{array}$ & \\
\hline $\begin{array}{l}\text { Relations } \\
\text { entre firmes }\end{array}$ & $\begin{array}{l}\text { Petites } \\
\text { et grandes firmes } \\
\text { en concurrence }\end{array}$ & $\begin{array}{l}\text { Externalisation } \\
\text { des grandes } \\
\text { vers les petites }\end{array}$ & $\begin{array}{l}\text { Coopération/ } \\
\text { concurrence } \\
\text { horizontale } \\
\text { coopération verticale }\end{array}$ & \\
\hline
\end{tabular}


Le mode d'organisation du district industriel, qui permet de différencier les formes III et IV souffre d'ailleurs discussion, car les districts ne paraissent présenter ni direction, ni structure hiérarchisée. Tout au plus peut-on discerner l'existence de «firmes-guides» assumant, par exemple, un rôle coordinateur dans le domaine de la distribution du produit, ou du design au niveau de sa conception. Il convient toutefois de souligner le fait que ce rôle d'impulsion n'est pas toujours joué par la même firme à travers le temps dans un district déterminé.

C'est la raison pour laquelle l'interventionnisme (étatique? local?) apparaît comme une nécessité pour pallier l'absence de centre de décisions stratégiques; c'est sur ce point essentiel aux yeux des auteurs (Brusco, 1990) que repose la distinction entre les districts industriels de type 1 et 2 .

Ces premières approches mettent l'accent sur la nécessité de tenir compte, dans l'approche en termes de districts industriels, simultanément :

- de la nature et de la diversité des relations entre petites entreprises ;

- de l'impact de l'orientation des relations petites entreprises - grande entreprise sur l'organisation du district ;

- du mode d'organisation du local.

\section{2. ... aux typologies du développement local}

Certains auteurs, considérant comme « trop générale » l'expression de district industriel, préfèrent situer leurs essais de typologie dans le cadre des systèmes localisés de petites entreprises. Ainsi procède G. Garofoli (1989) lorsqu'il propose une typologie basée sur l'évolution des districts industriels en Italie. La différence d'avec l'approche plus historique de Brusco se situe dans la recherche en tant que base de la typologie d'une logique d'organisation des relations entre petites entreprises localisées. Cet auteur distingue ainsi trois degrés :

- aire de production spécialisée (type 1);

- système productif local (type 2);

- aire-système (type 3),

traduisant une complexité croissante de l'organisation interne du système, en même temps qu'une rationalisation élargie quant aux relations avec d'autres systèmes. Pour illustrer ce propos, nous esquisserons deux schémas comparatifs des systèmes de petites entreprises traités par Garofoli, du double point de vue des structures de production d'une part, et des relations aux autres systèmes d'autre part. 


\section{Structures de production}

\section{TABleau 2}

\section{Structures de production}

\begin{tabular}{c|c|c|c}
\hline \multirow{2}{*}{$\begin{array}{c}\text { Organisation } \\
\text { de la production }\end{array}$} & $\begin{array}{c}\text { Aire de production } \\
\text { spécialisé }\end{array}$ & $\begin{array}{c}\text { Système } \\
\text { productif local }\end{array}$ & Aire-système \\
\cline { 2 - 4 } & Horizontale & Verticale & Spécialisée \\
\hline Produit & Même produit & $\begin{array}{c}\text { Différentes phases } \\
\text { de la production } \\
\text { du même produit }\end{array}$ & Différents secteurs \\
\hline $\begin{array}{c}\text { Échanges } \\
\text { inter-industriels }\end{array}$ & Peutions & $\begin{array}{c}\text { Relations } \\
\text { intra-industrielles }\end{array}$ & $\begin{array}{c}\text { Relations } \\
\text { inter industrielles }\end{array}$ \\
\hline Concurrence & $\begin{array}{c}\text { Coopération/ } \\
\text { Concurrence } \\
\text { Complémentarité }\end{array}$ & $\begin{array}{c}\text { Interrelations } \\
\text { entre P.E. } \\
\text { des différents } \\
\text { secteurs/ } \\
\text { partenariat } \\
\text { concurrence }\end{array}$ \\
\hline Technologie & Pas ou peu & $\begin{array}{c}\text { Diffusion } \\
\text { connaissances } \\
\text { technologiques }\end{array}$ & $\begin{array}{c}\text { Contrôle } \\
\text { de la technologie }\end{array}$ \\
\hline
\end{tabular}

L'élément essentiel dans l'évolution des structures de la production, selon Garofoli, réside dans le contrôle de la frontière technologique.

Dans le système de type 1 , on ne trouve pas trace de technologie mais il existe des savoir-faire qui traduisent en fait le passage d'un système artisanal au système industriel. Le système 2 utilise des technologies, parfois même assez complexes, et la diffusion des nouvelles technologies s'effectue grâce à la proximité entre les entreprises appartenant au même système productif, notamment à travers la «circulation» entre les entreprises de la main-d'œuvre qualifiée.

Le système 3 «endogénéise » la technologie, ce qui permet un accroissement de la productivité des entreprises, de leur efficience. En revanche, l'emploi industriel n'augmente pas : ce qui pourrait conduire, à la suite d'une analyse superficielle, à traduire le passage du type 2 au type 3 comme une déstructuration du système et/ou comme un manque de spécialisation. En effet, on ne retrouve pas, en type 3 , les articulations en chaîne existant entre les différents stades de la production d'un produit caractéristiques du type 2. Mais il s'agit en fait d'une spécialisation accrue dans certains stades stratégiques de la 
production: nous pouvons parler d'une spécialisation stratégique pour la différencier d'une spécialisation productive.

\section{Relations aux autres systèmes}

Les stratégies d'externalisation poursuivies par les grandes entreprises ont pour conséquence une déconcentration de la production : c'est la situation du système 1 (tableau 3).

Dans le système 2, il est possible, en franchissant un degré dans la complexité du système, de parler de décentralisation de la production. En effet, l'organisation de la production d'un bien est assurée par le système de petites entreprises. C'est pourquoi on peut même dire que c'est là que s'opère la mâ̂trise d'un secteur de production. Cette progression est liée à l'introduction de nouvelles technologies, au travers desquelles les petites entreprises, plus performantes, peuvent rééquilibrer les relations entretenues avec les grandes entreprises. Dans des situations de sous-traitance, on passe de la sous-traitance de capacité à la sous-traitance de spécialité, voire d' «intelligence». Les relations d'échanges entre grandes et petites entreprises, d'asymétriques, évoluent vers la symétrie, visant un partenariat.

TABLEAU 3

Relations aux autres systèmes

\begin{tabular}{c|c|c|c}
\hline \multirow{2}{*}{$\begin{array}{c}\text { Relation } \\
\text { au système }\end{array}$} & $\begin{array}{c}\text { Aire de production } \\
\text { spécialisée }\end{array}$ & $\begin{array}{c}\text { Système productif } \\
\text { local }\end{array}$ & Aire - système \\
\cline { 2 - 4 } & $\begin{array}{c}\text { Déconcentration } \\
\text { de la production }\end{array}$ & $\begin{array}{c}\text { Division du travail } \\
\text { Spécialisation } \\
\text { Maîtrise d'un secteur }\end{array}$ & $\begin{array}{c}\text { Maîtrise } \\
\text { d'une filière }\end{array}$ \\
\hline au système local & $\begin{array}{c}\text { Constitution } \\
\text { de «zones } \\
\text { industrielles » } \\
\text { Relation entre } \\
\text { système institutionnel } \\
\text { local et système } \\
\text { productif local } \\
\text { Demande } \\
\text { d'une politique } \\
\text { d'intervention }\end{array}$ & $\begin{array}{c}\text { Système } \\
\text { autorégulé }\end{array}$ \\
\hline «Culture & Non & $\begin{array}{c}\text { Oui } \\
\text { centrée }\end{array}$ & $\begin{array}{c}\text { Oui } \\
\text { système » }\end{array}$ \\
\hline
\end{tabular}

Le système 3 préside au passage à un stade supérieur, dans lequel les petites entreprises peuvent «produire» la technologie, modifier ou créer des 
processus technologiques. Dès lors, la maîtrise de la filière de production à partir de la maîtrise de points stratégiques de la filière devient possible. La spécialisation n'est pas réalisée sur chaque stade de l'élaboration d'un produit, mais bien sur des points déterminants. Garofoli parle, à propos de ce type 3 de système "autocentré»; nous préférons l'expression autorégulé, car il ne s'agit évidemment pas d'un modèle autarcique, mais au contraire d'un système très ouvert, capable d'internaliser les apports et connaissances extérieurs.

La présence de ce que nous nommons une «culture - système» (à l'instar de la culture entreprise) est déterminante dans la structuration et la spécification du système localisé. Lorsque cette culture existe, les relations entre système productif local, système institutionnel et système social se renforcent et se fondent en symbiose. Cette «culture - système » peut être assimilée à la notion de «milieu », telle que la conçoit Denis Maillat (1990), l'illustrant par l'exemple de l'Arc Jurassien (1991). Selon cet auteur, le milieu renvoie à un ensemble cohérent appuyé et articulé autour de trois éléments :

- un système territorialisé de production ;

- un ensemble de savoir-faire, une « culture technique »;

- des acteurs en interaction réciproque.

Le milieu est ainsi défini comme processus de perception, de compréhension et d'actions continuelles. Encore convient-il de remarquer que le milieu ne coïncide pas strictement avec un territoire géographique et/ou industriel déterminé : il peut déborder ce territoire, ou à l'inverse être inscrit à l'intérieur d'une frontière plus restreinte. C'est la raison pour laquelle nous avons choisi de nous référer à l'expression «culture - système », dont la présence suppose un ensemble de relations interentreprises, d'abord, mais aussi sociales, des échanges d'informations - et de formation - des synergies et collaborations industrielles et institutionnelles.

La distinction introduite entre culture - système «centrée » sur le niveau local et «excentrée» de l'aire - système vise à souligner le fait que dans l'airesystème, les acteurs locaux sont capables de faire jouer des synergies entre le système localisé et des éléments appartenant aux systèmes national et international.

En guise de conclusion d'étape, nous soulignerons le fait que la notion de district industriel, en tant que degré dans des typologies tend à disparaître. Mais elle doit être retenue quasi systématiquement lorsque l'on cherche à comprendre le mode de structuration et de développement des systèmes productifs localisés de petites entreprises.

Ainsi, le développement local, même appuyé sur de petites unités, ne peut se réduire au seul modèle du district industriel. 


\subsection{Le district : mode ou modèle ?}

\section{Qu'est-ce que le " modèle » du district industriel ?}

Au-delà des définitions données, les auteurs se rejoignent sur quatre points :

La spécialisation flexible: (Piore,1990; Capecchi, 1990) traduit les capacités d'adaptation et d'innovation des firmes du district, ainsi que leur aptitude à répondre aux modifications rapides de la demande. Ces atouts reposent sur la flexibilité de la force de travail et des réseaux de production, induite par une accumulation localisée de savoir-faire et d'aptitudes spécialisées.

Une spécificité organisationnelle: l'organisation et le leadership sont le fait de petites firmes, souvent à propriété familiale, reliées entre elles et s'articulant selon une division spécialisée. Chaque groupe d'établissements se spécialise dans un stade particulier de la production et apparaît complémentaire des autres dans le district: il existe donc une sorte d'interdépendance «organique». L'ensemble du système de petites entreprises apparaît ainsi apte à réaliser des économies externes.

Des relations interfirmes de conflit/coopération: l'ensemble des petites entreprises du district ne correspond pas à une simple juxtaposition d'unités productives, mais bien à un système organisé, impliquant des relations spécifiques interentreprises. Une caractéristique essentielle du district industriel réside dans des formes diversifiées de coopération interfirmes. Mais cette coopération (Brusco, 1990) est couplée avec une concurrence : la concurrence interviendrait entre firmes produisant le même produit, ou situées au même stade de l'élaboration d'un produit donné. La coopération se nouerait surtout dans le domaine de l'innovation technologique entre firmes situées à des niveaux de production différents, ou à travers les relations interindustrielles.

Un système globalisé : le district doit être conçu comme un tout. Ainsi, d'étroites relations existent entre les différentes sphères économique, sociale et politique, et le fonctionnement de l'une d'entre elles est déterminé par le fonctionnement et l'organisation des autres. L'interaction entre le niveau économique et le niveau social représente un trait remarquable du district industriel. On se trouve en présence d'un système particulier de valeurs permettant d'ordonner et de réguler l'organisation sociale des districts (Courlet, 1992).

Ces caractéristiques semblent ainsi déterminer un modèle «idéal» de district industriel. Mais ce schéma ne doit pas être confondu avec un mode idéal du développement local. En fait, le district industriel ne doit être considéré «ni comme un mythe, ni comme un interlude» (Trigilia, 1990), mais comme une forme particulière du développement localisé. 


\section{Les limites du " modèle » de district industriel}

Nous déterminerons deux limites :

1. Le district industriel constitue une forme particulière de développement local appuyé sur des petites entreprises, mais il ne doit pas être assimilé à un modèle idéal et unique de système productif localisé. Certes, le district industriel peut apparaître comme un mode d'organisation efficient de système productif localisé, et représenter à ce titre un objectif à atteindre pour les responsables des politiques de développement local, désireux de «valoriser » leur territoire. Mais il faut bien souligner que le district industriel ne constitue qu'un mode, parmi bien d'autres, du développement local, quand bien même ce développement implique la mise en œuvre d'une politique appuyée sur de petites entreprises.

2. Le district industriel ne représente pas un «passage obligé » lorsque l'on se place dans la perspective du développement d'ensembles de petites entreprises localisées. Car le district exige un certain nombre de conditions en l'absence desquelles on ne peut concevoir une telle forme de développement. Notons-les brièvement :

- Il suppose la présence d'une ou de deux activités industrielles particulières auxquelles prennent part l'ensemble des firmes, aux différents stades du processus de production.

- Il exige ce qu'Alfred Marshall appelait une " atmosphère industrielle », qui réfère aux multiples relations entre les entreprises du district, sans supposer pour autant une fermeture vis-à-vis de l'environnement national et international.

- Il détermine la formation d'un milieu, symbiose complexe d'éléments économiques et sociaux, supposant en tout état de cause une histoire industrielle et sociale.

Par conséquent, le district industriel apparaît comme un mode particulier d'organisation des systèmes productifs localisés; il ne doit en aucun cas être pris comme un modèle idéal et unique à appliquer dans l'élaboration d'une politique de développement territorial. Toutefois, il doit être considéré comme un concept analytique (ce n'est pas le seul) pour l'étude de la structuration et du développement des systèmes productifs locaux.

Nous allons ainsi tenter de l'utiliser en tant que référent conceptuel dans l'étude de l'aire de Montpellier. 


\section{L'aire de Montpellier : un district industriel ?}

La question posée ici, de manière d'ailleurs quelque peu provocatrice, consiste à estimer l'impact d'une politique d'intervention menée par les autorités locales sur le développement d'un territoire déterminé - en l'occurrence, l'aire de Montpellier (France) - en analysant cette démarche en référence au concept de district industriel.

Il convient en premier lieu de rappeler les caractéristiques locales, avant de préciser les éléments de la politique mise en œuvre.

\subsection{Quel système productif local ?}

Lorsque l'on s'intéresse à l'analyse des systèmes productifs locaux, deux formes organisationnelles sont à considérer simultanément : l'organisation productive et l'organisation territoriale.

\subsubsection{Les caractéristiques productives}

Trois caractéristiques déterminent la spécificité de cette aire :

\section{Un tissu économique sous-industrialisé}

- L'étude du secteur secondaire fait apparaître le poids très important du bâtiment par rapport aux activités manufacturières : il représente en effet $52 \%$ des établissements du secteur secondaire, mais seulement $32 \%$ des salariés, contre respectivement $48 \%$ et $68 \%$ pour l'industrie (Gapillout, 1990).

- L'ensemble des seules activités manufacturières traduit une très grande hétérogénéité de l'industrie ; les industries agro-alimentaires ne sont pas particulièrement représentées, alors que l'environnement régional est assez fortement marqué par l'agriculture.

\section{Un tissu économique tertiarisé}

La part très remarquable des services est une constante dans l'aire de Montpellier. Ainsi, l'industrie (y compris le bâtiment) regroupe $14 \%$ des établissements et $35 \%$ des salariés ; les services, respectivement $30 \%$ et $30 \%$; le commerce compte $56 \%$ des établissements, mais $34 \%$ seulement des salariés.

Une étude plus affinée des activités tertiaires (hors commerce) laisse apparaître l'importance des services rendus aux entreprises, ce qui souligne la particularité, voire le paradoxe d'un tissu économique par ailleurs peu industrialisé. 


\section{Un tissu économique atomisé}

Les entreprises sont, dans leur grande majorité petites et jeunes. Le poids des petites entreprises $(\mathrm{PE})$ - moins de 50 salariés -, et des très petites entreprises (TPE) - de 1 à 10 salariés - est très important : les TPE représentent $73 \%$ des établissements industriels, les PE (11 à 49 salariés), $22 \%$. Les pourcentages sont encore plus élevés dans les services : respectivement 87 et $11 \%$.

Cette atomisation du système productif est liée au fait qu'on perçoit une accélération de la création d'entreprises, tout particulièrement entre 1985 et 1989. D'où la «jeunesse » des entreprises implantées dans l'aire de Montpellier: on peut considérer que la moitié du tissu économique s'est constitué au cours des 5 à 7 dernières années. Ce particularisme suppose une certaine turbulence: on constate un nombre élevé de disparitions d'entreprises récemment créées, même si le taux de couverture des fermetures par les ouvertures reste largement positif, du moins jusqu'en 1991.

L'aire de Montpellier apparaît ainsi quelque peu atypique du point de vue du système productif, en tout état de cause, assez éloignée du cadre déterminé par le district industriel. Qu'en est-il en ce qui concerne l'organisation territoriale?

\subsubsection{Les caractéristiques territoriales}

Le caractère atypique transparaît également lorsque l'on se place du point de vue territorial. Il s'agit en effet d'un :

\section{Territoire "à construire »}

Paradoxalement, on peut dire que le local n'existe pas sur ce territoire. L'aire de Montpellier ne présente ni tradition industrielle, ni cohérence sociale.

L'aire n'a pas de tradition industrielle, donc pas d'atmosphère industrielle: on ne peut parler de bassin d'emploi, ni de savoir-faire spécifique. De même, aucune activité industrielle majeure n'émerge dans le tissu productif.

L'aire ne présente pas non plus de cohérence sociale. Pendant longtemps l'environnement régional était caractérisé par la dominante agricole (monoculture viticole), puis à la suite d'opérations d'aménagement du territoire dans les années 60, par le tourisme. Même si la population est nombreuse, en accroissement, aucune «communauté» ne se dégage ; il n'existe pas de «culture région ».

Toutefois, si le local ne se décrète pas (Planque, 1991), il peut néanmoins se construire. C'est bien là l'objet de l'intervention des gouvernements territoriaux. 


\section{Territoire du Sud}

Ce qui apparaît une évidence peut être précisé à travers les représentations des acteurs locaux et les positionnements nationaux et européens recherchés (Leclerc, 1991). La non-appartenance à la " dorsale européenne » mise en évidence par les géographes - axe reliant Londres à Milan - privilégiant l'Est du territoire national, semble ainsi constituer un handicap pour le Sud. Dans cet environnement européen, l'aire de Montpellier peut alors apparaître comme périphérique, donc en position défavorisée. Toutefois, point de passage obligé entre l'Italie et l'Espagne, débouché des voies de communication vers l'Europe du Sud, elle peut envisager une stratégie de positionnement par rapport à l'arc méditerranéen.

\section{Territoire urbain}

En tant que tel, l'aire de Montpellier offre les services supérieurs caractéristiques des villes. Universités et services de recherche y sont fortement représentés, ce qui constitue autant de «facteurs élitistes de la modernité territoriale » (Leclerc, 1991). Encore faut-il valoriser ces facteurs : c'est bien le but d'une politique territoriale.

\subsection{Quelle politique de développement ?}

\subsubsection{Le cadre de la stratégie}

Au regard du référent conceptuel évoqué, le district industriel, l'aire de Montpellier, semble présenter une structure bien dissemblable de cette forme d'organisation.

Du point de vue de l'organisation productive, la condition d'un ensemble de petites entreprises est respectée. Mais il faut souligner l'absence d'une activité industrielle majeure, et donc l'impossibilité de trouver un savoir-faire ou des aptitudes particulières de la main-d'œuvre.

Si les petites entreprises sont nombreuses, les interrelations de conflit/ coopération caractéristiques n'apparaissent pas en tant que trait remarquable. Tout au plus peut-on voir se tisser quelques relations privilégiées entre petites entreprises et petites entreprises de services, bien insuffisantes pour constituer un réseau dense de relations.

Du point de vue de l'organisation territoriale, l'absence de tradition industrielle et sociale signifie que la cohérence locale ne peut s'appuyer sur un fait local puisant ses sources dans l'histoire. Il est impossible de tirer des avantages d'un effet spontané de milieu. Ici, le local est à construire, et cette construction ne peut venir que de la politique de développement induite par les autorités locales. 
Pourtant, ce territoire présente des atouts qui peuvent servir de base à la structuration d'un système productif cohérent:

- en premier lieu, la présence de nombreuses petites entreprises, jeunes, très insérées dans le tissu local ;

- en second lieu, la ville de Montpellier dont l'image attractive joue un rôle majeur. Les services urbains, les infrastructures, les voies de communication, voire le fait culturel, constituent un environnement socio-économique favorable ;

- enfin, le potentiel scientifique et de recherche représente un levier d'action tout à fait essentiel.

Tous ces éléments ne correspondent pas véritablement au cadre de référence tracé par le district industriel. Il n'en reste pas moins qu'ils constituent autant d'atouts pour une stratégie de développement du territoire.

\subsubsection{La démarche stratégique}

Compte tenu du cadre dans lequel doit s'inscrire une politique de développement de l'aire de Montpellier, il est possible de synthétiser de la façon suivante la démarche stratégique (Marchesnay, 1986).

Les buts: ce sont les objectifs du gouvernement territorial concerné, à savoir la Ville de Montpellier, ainsi que les communes de la «couronne», ce que l'on nomme le «district de Montpellier» (dans un sens administratif).

L'objectif vise à dynamiser le tissu économique. Compte tenu de l'environnement local, à savoir les atouts et les faiblesses énoncés ci-dessus, la dynamisation et le développement local passent par la création d'un territoire technopolitain.

Au-delà du souci de création d'emplois et de renforcement d'un tissu économique sans tradition industrielle, l'objectif stratégique vise à positionner l'aire de Montpellier dans l'environnement concurrentiel des métropoles européennes.

Les choix stratégiques : un double choix apparaît :

- faire jouer les synergies entre acteurs locaux ;

- renforcer les réseaux de petites entreprises.

Ces deux volets sont d'ailleurs liés, car il s'agit d'établir des connexions entre acteurs locaux : entreprises, laboratoires de recherche et universités. Mais compte tenu du caractère atomisé du tissu économique, la connexion entre services supérieurs et entreprises - malgré la présence de quelques grands établissements - doit nécessairement passer par le branchement des petites 
entreprises sur les réseaux visant à faire jouer des effets de synergie. Il convient donc de renforcer les petites entreprises, de les structurer en réseau, afin d'obtenir un système de petites firmes cohérent, susceptible de constituer une sorte de «masse critique».

Les moyens: nous présenterons ici un outil d'intervention visant à concilier le double choix stratégique énoncé. On comprendra aisément que, par ailleurs, d'autres actions soient menées pour bâtir et développer une technopole.

L'outil concerné est le Centre européen d'entreprise et d'innovation (CEEI) Cap Alpha. L'un des problèmes majeurs, lorsque l'on considère un ensemble de petites entreprises, tout particulièrement de jeunes entreprises, est celui de leur pérennité. Or, dans les régions où se créent de nombreuses petites entreprises, l'intervention locale est longtemps restée limitée à la seule aide à la création. Ce type de politique, s'il favorise certes dans un premier temps l'émergence de nouvelles entreprises et donc la création d'emplois, ne témoigne pas nécessairement d'une vision stratégique à un terme plus long. En effet, il convient non seulement d'aider à la création, mais encore d'accompagner l'activité d'entreprise, afin d'organiser un ensemble de petites entreprises en un système cohérent. C'est dans ce but que l'opération Cap Alpha été lancée à Montpellier en 1987: conçue au départ comme une pépinière d'entreprises, elle a ensuite évolué pour prendre, à la fin de l'année 1988, la forme d'un centre européen d'innovation.

\subsubsection{L'outil stratégique d'intervention}

\section{L'outil}

Cap Alpha peut être d'abord considéré, comme une forme d'aide à la création d'entreprises, pour les « accompagner » au cours des deux premières années de démarrage, après avoir éventuellement préparé le dossier de création dans la phase d'incubation.

Cap Alpha bénéficie du statut européen de Centre d'entreprise et d'innovation (Business Investment Centers) qui permet d'offrir aux entreprises innovantes une structure de services, essentiellement de «matière grise »; à la différence d'une pépinière qui est avant tout un bâtiment offrant des services matériels à de jeunes entreprises. Ainsi sont proposés des services de formation, préparation du plan d'affaire, aide au développement technologique, conseils en gestion, immobilier, etc.

Nous avons mentionné plus haut la place importante occupée par les entreprises de services rendus aux entreprises (SRE) dans le tissu économique local, ces dernières prenant d'ailleurs le plus souvent la forme de très petites firmes. 
La présence de ces petites entreprises de SRE dans l'aire de Montpellier constitue un atout pour les responsables de Cap Alpha, en leur donnant la possibilité d'organiser des relations avec les entreprises en démarrage. La jeune entreprise hésite en effet à recourir à une entreprise de services, tout particulièrement lorsqu'il s'agit de conseil. La raison en est double: d'une part, le coût, quasi inenvisageable pour une entreprise en démarrage en termes de coût d'opportunité ; d'autre part, l'incertitude quant à la qualité de la prestation. Cap Alpha apparaît ainsi comme «acteur-relais » dans la relation entre petite entreprise en démarrage et petite entreprise de services; il représente la garantie d'une prestation choisie et adaptée, en «agréant » certaines petites firmes de services, et il assure une partie du financement du conseil.

En ce qui concerne la sélection des entreprises - ou des projets - admises à Cap Alpha, l'exigence d'innovation est majeure, qu'il s'agisse de la technologie, sans qu'elle soit nécessairement de pointe, du produit ou du processus, ou d'une idée de marché (tableau 4).

\section{TABLEAU 4}

\section{Stratégie de développement du territoire : l'expérience Cap Alpha}

\begin{tabular}{|c|c|c|c|}
\hline $\begin{array}{l}\text { Objectifs de } \\
\text { l'intervention locale }\end{array}$ & \multicolumn{3}{|c|}{$\begin{array}{l}\text { Création d'une activité économique motrice } \\
\text { Recherche de valeur ajoutée sur le plan local }\end{array}$} \\
\hline \multirow{2}{*}{ Axe déterminant } & \multicolumn{3}{|c|}{$\stackrel{\downarrow}{\text { Stratégie TPM }}$} \\
\hline & $\underset{\text { Technologie }}{\nabla}$ & $\stackrel{\downarrow}{\nabla}$ & $\nabla_{\text {Marché }}^{7}$ \\
\hline $\begin{array}{l}\text { Profil } \\
\text { des entreprises }\end{array}$ & $\begin{array}{l}\text { Nécessaire, } \\
\text { mais pas } \\
\text { obligatoirement } \\
\text { high-tech }\end{array}$ & $\begin{array}{c}\text { Porteur } \\
\text { d'innovation } \\
\text { Produit ou } \\
\text { processus }\end{array}$ & $\begin{array}{l}\text { National } \\
\text { et/ou } \\
\text { international }\end{array}$ \\
\hline
\end{tabular}




\section{TABleau 5}

\section{Création d'entreprises : activités innovantes}

\begin{tabular}{c|c|c|c}
\hline & Informatique & Électronique & $\begin{array}{c}\text { Agro-alimentaire } \\
\text { Bio-industrie }\end{array}$ \\
\hline $\begin{array}{c}\text { En } \% \\
\text { des entreprises } \\
\text { créées }\end{array}$ & $32 \%$ & $21 \%$ & $25 \%$ \\
\hline
\end{tabular}

Le caractère innovant réside, dans la majorité des cas, dans le produit lui-même (Calleja et Thénaisie, 1990), (tableau 6).

TABLEAU 6

Création d'entreprises : types d'innovations

\begin{tabular}{c|c|c|c}
\hline & $\begin{array}{c}\text { Innovation } \\
\text { produit }\end{array}$ & $\begin{array}{c}\text { Innovation } \\
\text { processus }\end{array}$ & $\begin{array}{c}\text { Innovation } \\
\text { commerciale }\end{array}$ \\
\hline $\begin{array}{c}\text { En \% } \\
\text { des entreprises } \\
\text { créées }\end{array}$ & $72 \%$ & $7 \%$ & $21 \%$ \\
\hline
\end{tabular}

On constate donc la primauté de l'innovation technologique.

Il convient de souligner que, au-delà de la création de petites entreprises innovantes, Cap Alpha a développé le suivi d'entreprises existantes, porteuses elles aussi d'innovation: 115 entreprises ont ainsi été «accompagnées » au cours des trois dernières années.

Le faible taux d'échec - 7 entreprises aidées ont cessé leurs activités tient à la forte sélection à laquelle étaient soumis les projets. Il peut également être imputé au fait de privilégier deux à trois secteurs d'activité seulement, organisant ainsi une cohérence dans le maillage du tissu économique local.

Une vision stratégique?

On peut s'interroger sur le caractère stratégique de l'outil que représente Cap Alpha. En effet, l'action de ce Centre européen d'entreprise et d'innovation (CEEI) peut sembler très ponctuelle, voire marginale par rapport au but poursuivi par la politique de développement territorial, c'est-à-dire la création d'un territoire technopolitain. Pour ce faire, la technopole de Montpellier met l'accent sur cinq «Pôles d'excellence » pour lesquels elle dispose d'un « savoir-faire capital » en matière de recherche et d'activités secondaires ou tertiaires (Marchesnay, 1991) : 
- pôle Euromédecine (bio-médical, recherche pharmaceutique);

- pôle Agropolis (agro-industrie tropicale et méditerranéenne);

- pôle Informatique (robotique et intelligence artificielle);

- pôle Antenna (image et télécommunications ; câblage);

- pôle Héliopolis (tourisme, culture, congrès).

La stratégie technopole vise à constituer un réseau de transfert de technologies, par la création et l'accueil d'entreprises en faisant jouer des synergies entre le tissu des entreprises innovantes et les atouts locaux en matière de recherche.

Le problème majeur réside ici dans le fait que le tissu économique local, ainsi que nous l'avons décrit, apparaît sous-industrialisé, parcellisé, ne traduisant aucune cohérence orientée par un secteur moteur, alors même que les activités de recherche et de formation considérées comme atouts déterminants sont présentes depuis fort longtemps dans l'aire de Montpellier. Cela signifie donc que le système productif local «traditionnel» composé essentiellement de petites unités est apparu incapable d'accueillir et de diffuser l'innovation, en participant aux transferts de technologie.

Dès lors, quelle vision stratégique alternative apparaît? Il n'existe pas, localement, de grandes entreprises susceptibles de piloter ou de relayer la stratégie-technopole : la présence, depuis 25 ans dans l'aire de Montpellier, d'un établissement IBM important a contribué certes à l'apparition d'un réseau de sous-traitants, mais sans que celui-ci constitue un élément structurant du système productif local.

Le seul choix stratégique possible consiste donc, comme nous l'indiquions plus haut, à organiser le développement local à partir de réseaux cohérents de petites entreprises, orientées autour des activités majeures définies par les pôles. Si quelques petites unités œuvraient auparavant à l'intérieur du tissu «traditionnel » dans ces activités, leur nombre, leur taille et leur capacité innovatrice étaient trop limités pour constituer un point d'ancrage de la stratégie d'un territoire technopolitain.

D'où la nécessité de mettre en place un outil visant à créer et accueillir des petites entreprises innovantes dans les activités pouvant bénéficier des transferts de technologie et des synergies issus des acteurs locaux.

De plus, il convient non seulement de créer de nouvelles petites entreprises, mais encore d'accompagner leur développement, et de les brancher sur les réseaux existants ou à renforcer : c'est bien l'objet du CEEI (Centre européen d'entreprise et d'innovation) Cap Alpha, ce qui justifie ainsi le qualificatif 
d'outil stratégique. S'il ne représente bien entendu que l'une des mesures de la politique de développement local mise en œuvre dans l'aire de Montpellier, il apparaît tout à fait important dans l'optique de l'objectif de cette stratégie: dynamiser le tissu économique local en créant un territoire technopolitain.

\section{Conclusion : Montpellier, vers un district technologique?}

À l'issue de ces développements, il est maintenant possible de répondre à la question formulée au départ : une politique de développement local peut-elle viser à mettre en place un district industriel ? Cet objectif étant ciblé parce que le district industriel apparaît comme un mode d'organisation efficient d'un système productif localisé appuyé sur des petites unités.

Il convient d'abord de poser deux limites à l'effet-district :

- En premier lieu, il ne convient pas d'établir une correspondance stricte entre l'existence d'ensembles de petites entreprises et l'application du modèle du district : ce n'est pas parce que l'on constate la présence de petites entreprises qu'il faut penser automatiquement en termes de district industriel.

- En second lieu, quel que soit l'intérêt du concept de district, il ne peut apparaître comme un modèle du développement local.

Malgré ces limites, son intérêt majeur est d'ordre conceptuel dans la mesure où il représente une aide à la compréhension des modes de structuration et d'évolution d'un système productif localisé.

En tant que référent, il met l'accent sur les éléments essentiels pour induire et dynamiser une politique de développement local, au premier rang desquels figure la nécessaire cohérence d'un système productif autour d'activités communes, mais aussi d'une culture commune.

Au regard de ces apports, on peut donc estimer qu'en l'état actuel des choses, l'aire de Montpellier ne constitue pas un district industriel, n'en présentant ni les conditions organisationnelles productives, ni les conditions organisationnelles territoriales.

Qu'en sera-t-il dans l'avenir? La politique de développement local poursuivie par les autorités territoriales vise à créer un territoire technopolitain. Parmi les objectifs retenus, certains sont communs avec les conditions d'existence d'un district, comme le tissu de petites entreprises, ou la présence d'une activité majeure, encore que l'aire de Montpellier cherche à appuyer son développement 
sur les secteurs particulièrement innovants, alors que les districts industriels sont le plus souvent structurés autour d'activités plus traditionnelles (textile, mécanique).

Mais d'autres dimensions de la stratégie territoriale s'éloignent de la référence-district: ainsi la volonté d'organiser des réseaux entre les acteurs du système, petites entreprises d'une part, services supérieurs d'autre part, pour la production, le transfert et la valorisation des technologies. De même, le choix de la stratégie-technopole tend à faire diffuser l'influence des synergies et des réseaux en dehors du territoire: la dimension locale de la politique de développement ne sous-entend pas le localisme. On pourrait alors dire, pour conclure, que ce qui est visé dans l'aire de Montpellier ressort plutôt de la construction d'un district technologique, qui semble représenter la «version de la fin du $20^{\mathrm{e}}$ siècle du district marshallien » (Pecqueur et Rousier, 1991). Le district technologique, nécessairement urbain, tire un avantage des relations territoriales entre acteurs locaux, pour être plus performant sur les marchés internationaux. Mais le processus de régulation doit être recherché dans une unité culturelle fondée, non sur la cohérence sociale, mais sur celle des formations et des technologies.

La politique du développement menée dans l'aire de Montpellier apparaît cohérente avec ce schéma; mais malgré la dynamique suscitée, le territoire reste encore très largement à construire.

\section{Bibliographie}

BRusco, S. (1988), « The idea of the industrial district : its genesis », International Institute for Labour Studies, Genève, juillet.

Brusco, S. (1990), «The idea of the industrial district : its genesis », dans : F. Pyke, G. Beccatini et W. Sengenberger (éds), Industrial districts and inter-firms co-operation in Italy.

CAlleja, I. et C. ThÉnAisie (1990), Bilan de l'action du CEEI Cap alpha, Mémoire de maîtrise en sciences économiques, Université Montpellier I.

CAPECCHI, V. (1990), «A history of flexible specialisation and industrial districts in Emilia Romagna », dans F. Pyke, G. Becattini et W. Sengenberger (éds), op. cit.

Courlet, C. (1992), « Le district industriel de la vallée de l'Arve : origines, fonctionnement et évolution récente », Rapport PIRTTEM Développement local et ensembles de $P M E$, juillet. 
Gapillout, J.F. (1990), La diversité du tissu économique régional, Mémoire de maîtrise en sciences économiques, Université Montpellier I.

GAROFOLI, G. (1989), « Histoire des districts industriels italiens », Journées du groupe Développement local et ensembles de PME, Lyon, décembre.

LECLERC, R. (1991), « Le sud de la France, un chaînon manquant ? », document DATAR.

MAILlat, D. et J.C. PERRIN (éds) (1990), Entreprises innovatrices et réseaux locaux, Paris, Eresa-Economica.

MAILlat, D. (1991), « PME et système territorial de production », dans C. Fourcade, éd., Petite entreprise et développement local, Paris, Eska.

MARCheSnAY, M. (1986), La stratégie, Paris, Chotard.

MARCHESNAY, M. (1991), «Les transferts de technologie de la région vers les PME : le cas du Languedoc-Roussillon », Économies et sociétés, $\mathrm{F} \mathrm{n}^{\circ} 32$.

Pecqueur, B. et N. Rousier (1991), «Les districts technologiques, un nouveau concept pour l'étude des relations technologies/territoires ", Colloque ASRDLF, Montréal, septembre.

PIORE, M. (1990), « Work, labour and action : work experience in a system of flexible production », dans F. Pyke, G. Becattini et W. Sengenberger, (éds), op. cit.

PlanQue, B. (1991), « Note sur la notion de réseau d'innovation », Revue d'Économie Régionale et Urbaine, $\mathrm{n}^{\text {os }} 3 / 4$.

Pyke, F., G. BeCattini et W. SEngenberger (éds) (1990), Industrial districts and inter-firms co-operation in Italy, International Institute for Labour Studies, Genève.

Pyke, F. et W. Sengenberger (1990), « Small firms industrial districts and local economic regeneration : research and policy issues », International Conference, IILS, Genève, octobre.

TRIGILIA, C. (1990), «Italian industrial districts : neither myth nor interlude », International Conference, IILS, Genève, octobre.

ZEITLIN, J. (1990), «Industrial districts and local economic regeneration : models, institutions and policies », International Conference, IILS, Genève, octobre. 